\title{
Phylogenetic and genomic analyses of the ribosomal oxygenases Riox1 (No66) and Riox2 (Mina53) provide new insights into their evolution
}

Katharina E. Bräuer ${ }^{1}$, Kevin Brockers', Jasmin Moneer ${ }^{2}$, Annette Feuchtinger ${ }^{3}$, Evi Wollscheid-Lengeling ${ }^{4}$, Andreas Lengeling ${ }^{4,5}$ and Alexander Wolf ${ }^{1 *}$ (i)

\begin{abstract}
Background: Translation of specific mRNAs can be highly regulated in different cells, tissues or under pathological conditions. Ribosome heterogeneity can originate from variable expression or post-translational modifications of ribosomal proteins. The ribosomal oxygenases RIOX1 (NO66) and RIOX2 (MINA53) modify ribosomal proteins by histidine hydroxylation. A similar mechanism is present in prokaryotes. Thus, ribosome hydroxylation may be a well-conserved regulatory mechanism with implications in disease and development. However, little is known about the evolutionary history of Riox1 and Riox2 genes and their encoded proteins across eukaryotic taxa.

Results: In this study, we have analysed Riox 1 and Riox2 orthologous genes from 49 metazoen species and have constructed phylogenomic trees for both genes. Our genomic and phylogenetic analyses revealed that Arthropoda, Annelida, Nematoda and Mollusca lack the Riox2 gene, although in the early phylum Cnidaria both genes, Riox 1 and Riox2, are present and expressed. Riox 1 is an intronless single-exon-gene in several species, including humans. In contrast to Riox2, Riox1 is ubiquitously present throughout the animal kingdom suggesting that Riox 1 is the phylogenetically older gene from which Riox2 has evolved. Both proteins have maintained a unique protein architecture with conservation of active sites within the JmjC domains, a dimerization domain, and a winged-helix domain. In addition, Riox1 proteins possess a unique $N$-terminal extension domain. Immunofluorescence analyses in Hela cells and in Hydra vulgaris identified a nucleolar localisation signal within the extended $\mathrm{N}$ terminal domain of human RIOX1 and an altered subnuclear localisation for the Hydra Riox2.

Conclusions: Conserved active site residues and uniform protein domain architecture suggest a consistent enzymatic activity within the Riox orthologs throughout evolution. However, differences in genomic architecture, like single exon genes and alterations in subnuclear localisation, as described for Hydra, point towards adaption mechanisms that may correlate with taxa- or species-specific requirements. The diversification of Riox1/Riox2 gene structures throughout evolution suggest that functional requirements in expression of protein isoforms and/or subcellular localisation of proteins may have evolved by adaptation to lifestyle.
\end{abstract}

Keywords: Ribosome, Ribosomal oxygenases, Fe(II) and 2-oxoglutarate dependent oxygenases, Hydroxylation, JmjC, Jumonji, Mina53, NO66, Single exon genes, Intronless retroposed gene copies

\footnotetext{
* Correspondence: alexander.wolf@helmholtz-muenchen.de

${ }^{1}$ Institute of Molecular Toxicology and Pharmacology, Helmholtz Zentrum München-German Research Center for Environmental Health, Ingolstädter Landstrasse 1, 85764 Neuherberg, Germany

Full list of author information is available at the end of the article
}

(c) The Author(s). 2018 Open Access This article is distributed under the terms of the Creative Commons Attribution 4.0 International License (http://creativecommons.org/licenses/by/4.0/), which permits unrestricted use, distribution, and reproduction in any medium, provided you give appropriate credit to the original author(s) and the source, provide a link to the Creative Commons license, and indicate if changes were made. The Creative Commons Public Domain Dedication waiver (http://creativecommons.org/publicdomain/zero/1.0/) applies to the data made available in this article, unless otherwise stated. 


\section{Background}

The central dogma of molecular biology has described gene expression as a straightforward process in which a gene is transcribed into mRNA followed by translation into a protein [1]. In recent years, a multitude of regulatory processes of gene expression have been identified on transcriptional and posttranscriptional levels. The mRNA itself can be subjected to regulatory events such as alternative splicing or RNA modifications $[2,3]$, but also mRNA translation rates or translation selectivity of individual mRNA molecules can vary [4]. Initially the ribosome was thought to be a rather unaltered ribonucleoprotein particle responsible for translation of any incoming mRNA into a polypeptide. However, recent research has shown that ribosomes are highly specific translational machineries that underlie complex regulatory mechanisms in order to meet physiological requirements in different cell types or throughout development [4]. 'Specialized' ribosomes can be generated by various mechanisms, including changes in ribosomal protein composition or posttranslational modifications, like methylation or phosphorylation [5]. Very recently, ribosomal oxygenases (ROXs) have been identified that modify ribosomal proteins by hydroxylation of amino acids [6-10]. The ROXs are a subgroup of the enzyme superfamily of $\mathrm{Fe}(\mathrm{II})$ and 2-oxoglutarate (2OG) dependent oxygenases (2OG oxygenases). All known human 2OG oxygenases catalyse transfer of molecular oxygen to a prime substrate, which can be either amino acids or nucleotides [11]. Due to the manifold set of their substrates, 2OG oxygenases have been discovered to have central roles in many cellular processes including epigenetic regulation of gene expression, control of transcriptional initiation, and regulation of alternative splicing [12-15]. The ROXs, which include the nucleolar protein NO66 (official new nomenclature symbol RIOX1) and the MYC-induced nuclear antigen MINA53 (new nomenclature symbol RIOX2), directly modify ribosomal proteins $[6,8,9]$. RIOX1 has been demonstrated to hydroxylate histidine $216(\mathrm{H} 216)$ in the ribosomal protein RPL8 (uL2), whereas RIOX2 hydroxylates H39 in RPL27a (uL15) [6] (Fig. 1a). 2OG-oxygenase-catalysed ribosome hydroxylation is conserved from bacteria to humans. The Riox1/Riox 2 counterpart in prokaryotes, ycfD, hydroxylates arginine (R) 81 in Rpl16 [6] (Fig. 1b). In addition, RIOX1 and RIOX2 share similarities in substrate

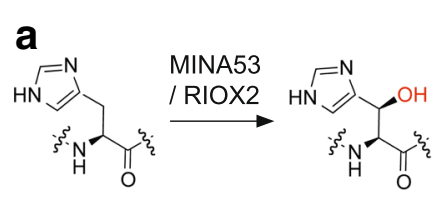

His

(RPL27A, human)

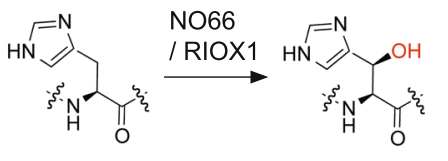

His (RPL8, human)

\section{b}

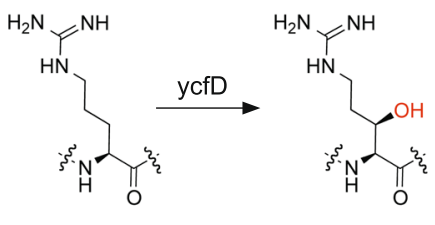

Arg (Rpl16, E.coli)
Arg-OH

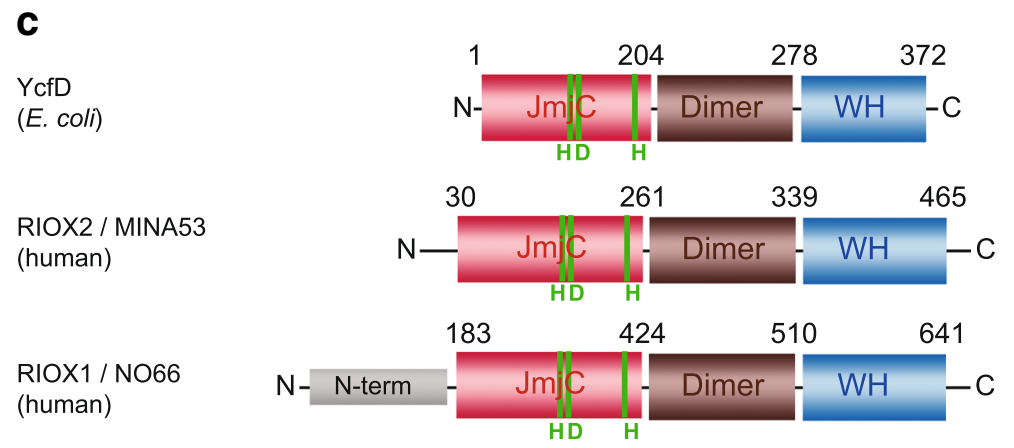

Fig. 1 The ribosomal oxygenases (ROXs) are a subgroup of Fe(II) and 2OG-dependent oxygenases that modify the ribosome and are present in pro- and eukaryotes. a The human ROXs RIOX2/MINA53 and RIOX1/NO66 hydroxylate histidine residues in the ribosomal proteins RPL27A and RPL8, respectively, whereas (b) the E.coli ycfD protein hydroxlyates an arginine in Rpl16 [6]. Protein sequence and crystal structure analyses confirmed a similar protein-domain architecture for the three proteins [16]. c They consist of a JmjC-domain (red), a dimerization domain (brown) for homo-oligomerization and a winged-helix $(\mathrm{WH})$ domain (blue). The aa triad HxD...H that coordinates the iron and is essential for catalytic activity is indicated in green (c) 
binding with ycfD and exhibit similar conserved protein domains [16]. Crystal structure analyses on recombinant enzymes revealed an $\mathrm{N}$-terminal Jumonji $\mathrm{C}$ $(\mathrm{JmjC})$ domain, which harbours the active site and the iron-coordinating residues, characteristic for all known $2 \mathrm{OG}$ oxygenases. A central dimerization-domain is responsible for homo-oligomerization in-vitro and the $C$-terminus contains a winged-helix (WH) domain [16] (Fig. 1c). Both, human RIOX1 and RIOX2 have been described to be involved in cancer cell growth. RIOX2 is a myc-target gene [17]. Its expression is upregulated in several cancers, including lung and breast cancer, and knockdown of RIOX2 in A549 cells inhibited cell proliferation [18, 19]. Elevated RIOX2 expression has been described in non-small cell lung cancers [20] and was reported to be associated with invasive colorectal cancer [21]. In addition, RIOX2 regulates immune responses as a transcriptional co-repressor of the interleukin-4 (Il4) encoding gene [22]. It has been described to polarize $\mathrm{T}$ helper 2 (Th2) cell responses in atopic pulmonary inflammation and to have a role in parasitic worm expulsion [23]. Riox1 is involved in osteoblast differentiation [24] and variations in its expression level have been reported to regulate skeletal growth and bone formation in mice $[25,26]$.

Here we provide the first comprehensive sequence analyses of the ribosomal oxygenases Riox1 and Riox2 in different eukaryotic species. We compared the domain architecture of both proteins and their exon-intron gene structures across a wide range of metazoan species. In addition we used immunofluorescence approaches to investigate expression in human cells and in the Cnidaria Hydra vulgaris.

\section{Results}

Evolutionary sequence and protein domain architecture conservation of the ribosomal oxygenases Riox 1 and Riox2 A sequence alignment of human RIOX2 (MINA53, 465 amino acids (aa)) and human RIOX1 (NO66, 641 aa) proteins revealed $23.2 \%$ identical and $16.4 \%$ similar aa (Fig. 2a). Both proteins exhibit the same protein domain structure, including a JmjC domain, a dimerization domain and a WH domain [16]. The RIOX1 protein has an $N$-terminal extension of 184 aa, which is absent in the RIOX2 protein (Fig. 2a). RIOX1 and RIOX2 are nuclear proteins with a strong accumulation in nucleoli $[27,28]$ (Fig. 2b,c). To identify Riox 1 and Riox 2 orthologs in other eukaryotic species we searched the genomes of the model organisms Mus musculus, Gallus gallus, Danio rerio, Caenorhabditis elegans and Drosophila melanogaster. An Ensembl genome browser search with the human RIOX1 protein sequence (Ensembl: ENSG000 00170468; Uniprot: Q9H6W3) revealed orthologous proteins in M. musculus, G. gallus, D. rerio, C. elegans and D. melanogaster. The $M$. musculus Riox1 protein sequence (Ensembl: ENSMUSG00000046791; Uniprot: Q9JJF3) showed $75.2 \%$ identity to the human sequence, with most sequence variation in the $\mathrm{N}$-terminal extension domain (Additional file 1: Figure S1). The G. gallus Riox1 protein (Ensembl: ENSGALG00000020454; Uniprot: Q5ZMM1) showed 49.3\% identity (Additional file 2: Figure S2), the D. rerio Riox1 43\% (Ensembl: ENSDA RG00000067838; Uniprot: A3KP59) (Additional file 3: Figure S3), the D. melanogaster Riox1 33.1\% (Ensembl: FBgn0266570; Uniprot: E2QD64) (Additional file 4: Figure S4) and the C. elegans Riox1 protein (Ensembl: WBGene00020902; Uniprot: O01658) $28.7 \%$ aa sequence identity (Additional file 5: Figure S5).

A search for Riox 2 sequences in the same set of organisms revealed no Riox2 orthologs in C. elegans and D. melanogaster. Searches with human RIOX2 (Ensembl: ENSG00000170854; Uniprot: Q8IUF8) or D. rerio Riox2 (Ensembl: ENSDARG00000036359; Uniprot: F1R7K2) sequence respectively revealed no homologous sequences, but detected the above described Riox 1 sequences for $C$. elegans and D. melanogaster as the sequences with highest homology.

Individual alignments of Riox2 sequences with the human RIOX2 showed 76.3\% identical aa for the $M$. musculus Riox2 sequence (Ensembl: ENSMUSG00000022724; Uniprot: Q8CD15) (Additional file 6: Figure S6), 70.8\% for the G. gallus Riox2 (Ensembl: ENSGALG00000039302; Uniprot: E1C6P1) (Additional file 7: Figure S7) and 57.2\% for the D. rerio Riox2 (Ensembl: ENSDARG00000036359; Uniprot: F1R7K2) (Additional file 8: Figure S8).

Genome sequence searches of additional invertebrate species from the Arthropoda, Annelida, Nematoda, and Mollusca clades also did not identify any putative Riox 2 orthologous genes (results discussed below), but the early metazoan animal Hydra vulgaris of the phylum Cnidaria exhibited both, Riox 1 and Riox2 sequences (Fig. 3). That prompted us to analyse the expression of the hydra orthologs in more detail.

\section{Characterization and cellular localization of hydra Riox1 and Riox2 proteins}

The fresh-water polyp Hydra is a pre-bilaterian animal of the phylum Cnidaria [29]. Database searches of the Hydra vulgaris genome [30] predicted two sequences encoding for Hydra Riox2 (HyRiox2) and Hydra Riox1 (HyRiox1). Amplification with corresponding primers, cloning and subsequent sequencing identified the predicted HyRiox2 (551aa) and HyRiox1 (628aa) protein sequences encoded in Hydra cDNAs. An alignment of these Hydra protein sequences with the human RIOX1 and RIOX2 proteins revealed 35.0 and $33.7 \%$ aa sequence identity, respectively (Additional file 9: Figure S9 and Additional file 10: Figure S10). A sequence comparison 


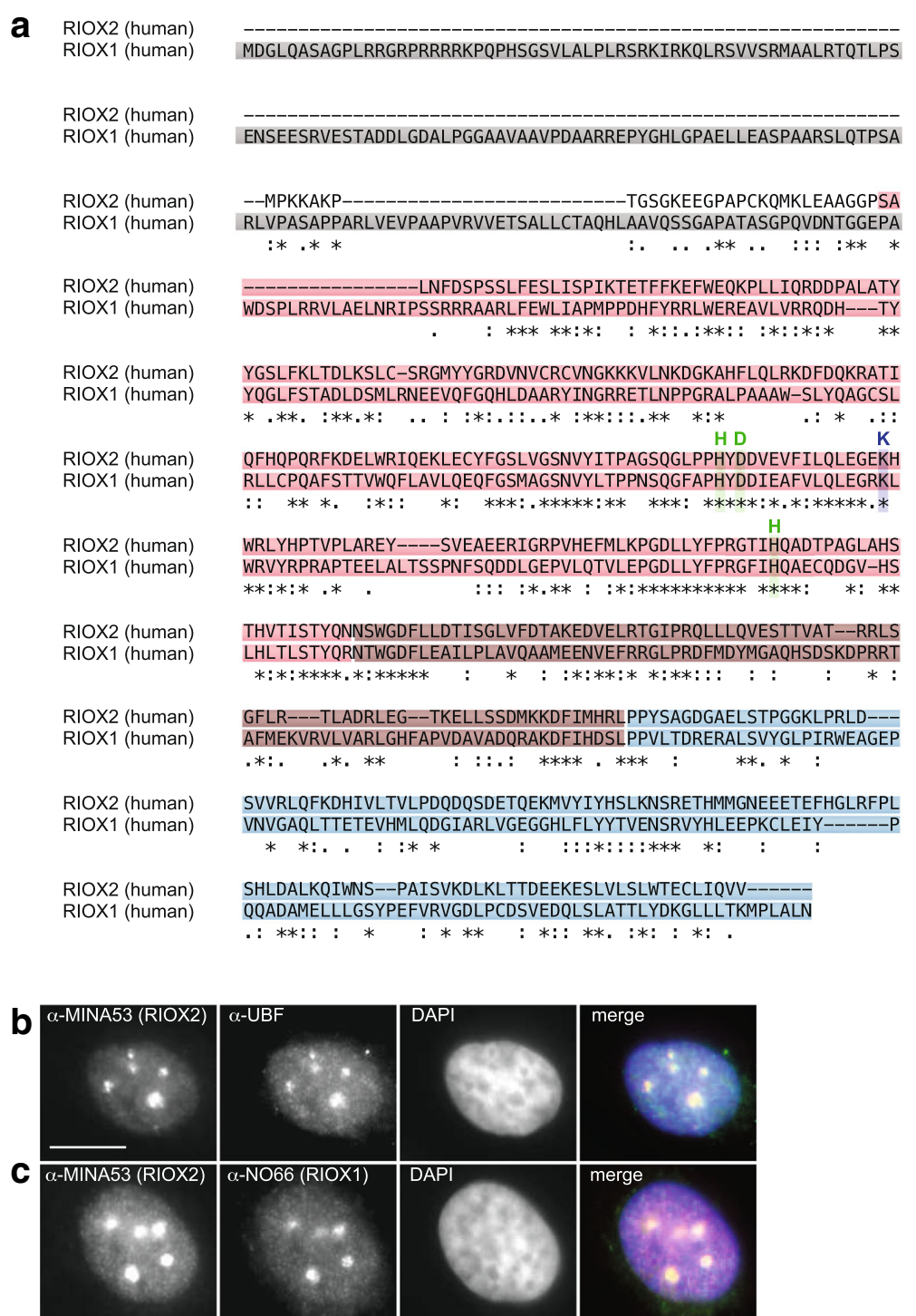

Fig. 2 Clustal omega sequence alignment [35] of human RIOX2 and RIOX1 aa sequences. a Protein domains are indicated in red (JmjC), brown (dimerization) and blue (WH). The RIOX1-specific N-terminal extension is marked in grey. The Fe(II) and 2OG-binding residues are highlighted in green or purple respectively. Both, human RIOX2 and RIOX1 localise to nucleoli. Immunofluorescent staining of endogenous RIOX2 (a-Mina53) in HeLa cells showed co-localisation with (b) the nucleolar marker protein upstream-binding factor 1 (a-UBF) and (c) with RIOX1 (a-NO66). Scalebar: 10 um

of the two identified Hydra ROXs showed, that HyRiox2 and HyRiox1 share 19.7\% identical aa (Fig. 3a). ROXs proteins are characterized by a double-stranded beta-helix fold (DSBH or JmjC domain) that harbours the active site, including residues for co-factor $(\mathrm{Fe}(\mathrm{II}))$ and co-substrate (2OG) binding [11]. Based on the crystal structures of human RIOX1 and RIOX2 proteins [16], both Hydra sequences exhibit the characteristic $\mathrm{Fe}(\mathrm{II})$ binding motif HxD/E...H (HyRiox2: H178xD180...H240; HyRiox1: H330xD332...H395) and also a conserved 2OG C5-carbo xylate-interacting lysine (K) residue (HyMina53: K193; HyNo66: K345) (Fig. 3a; Additional file 9: Figure S9, Additional file 10: Figure S10). Both Hydra homologs have also the characteristic ROX protein domain architecture with $\mathrm{JmjC}$, dimerization, and $\mathrm{WH}$ domains. In addition, the Hydra Riox1 homolog HyRiox1 exhibits an $\mathrm{N}$-terminal extension domain proximal to the JmjC domain. Interestingly, the HyRiox 2 contains two extended sequence segments in its dimerization domain and one extended sequence stretch in the $\mathrm{WH}$ domain. No corresponding sequences of these extensions are detectable in the human RIOX2 protein. The sequence extension in the HyRiox2 WH domain comprises a stretch of 64 additional residues, rich in the charged aa lysine $(K)$, aspartate (D) and glutamate (E) (42 of the 64aa) (Additional file 9: Figure S9). 

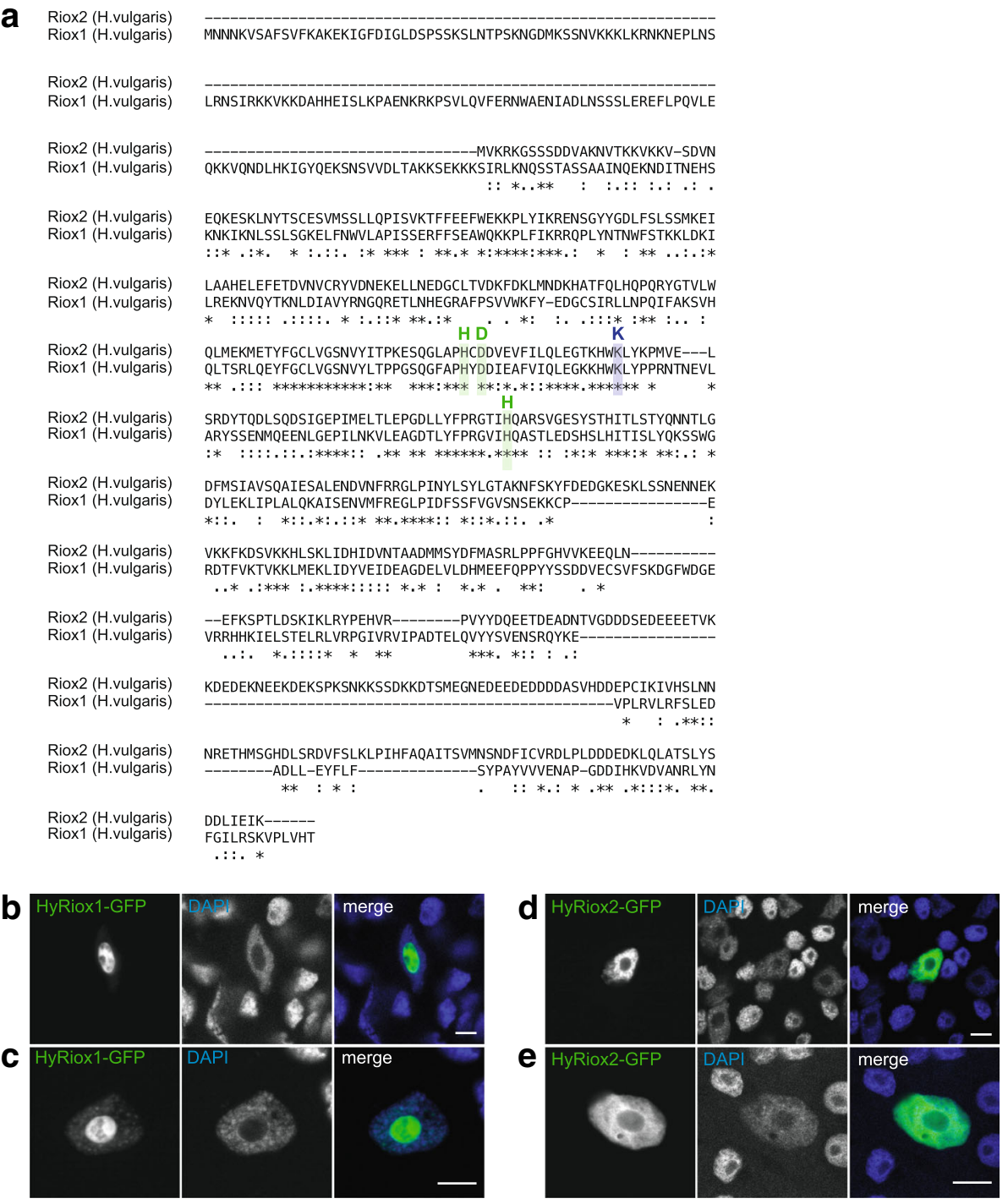

Fig. 3 Riox1 and Riox2 expression in Hydra. a Alignment of both Hydra sequences, with highlighted iron-binding motif (HxD ...H) (green) and predicted lysine-residue for 20G binding (blue). b, c Expression of GFP-tagged HyRiox1 in Hydra animals displayed nuclear localisation and strong accumulation in nucleoli. d, e HyRiox2 is also localised in nuclei, but an accumulation in nucleoli is not detectable. DNA-stain: DAPI. Scalebar: 10 um

To analyse the subcellular localization of HyRiox2 and HyRiox1, we expressed GFP-tagged full-length proteins in Hydra cells of intact animals after transfection with a particle gun [31]. Confocal imaging of ectopically expressed GFP-tagged HyRiox1 confirmed the nuclear localization of the protein with a prominent accumulation in nucleoli (Fig. 3b,c). Transfection of HyRiox 2 constructs revealed also expression in the nucleus; however, in contrast to the human RIOX2, HyRiox2 did not accumulate to nucleoli (Fig. 3d,e).

\section{Sequence analyses of Riox 1 and Riox2 proteins from different species}

The ROXs domain topology of homologous Riox 1 proteins is similar in all analysed species. The lengths of their JmjC domains (Riox1: 236-242aa), dimerization domains (Riox1: 86-93aa) and WH domains (Riox1: 129-133aa) are comparable in the analysed organisms, whereas the lengths of the $\mathrm{N}$-terminal extension varies from 87 in $D$. rerio to 286 aa in C. elegans (Additional file 11: Figure S11). An alignment of these Riox1 protein sequences showed strong conservation of the Fe(II) and 2OG-binding residues in the catalytic site of these enzymes (Additional file 11: Figure S11). The sequence homology implies a conserved function of Riox1 across species. Structural analysis of human RIOX1 co-crystallised with an Rpl8 peptide identified interactions of arginine (R) 297, tyrosine (Y) 328 and serine (S) 421 with the substrate peptide [16]. These residues are conserved in all Riox1 proteins across the analysed species (Additional file 11: Figure S11). Similarly, in 
Riox2 (Additional file 12: Figure S12), four primary amides (asparagine (N) 101, glycine (G) 136, G139, N165) and S257 in human RIOX2 have been identified to interact with its Rpl27a substrate peptide. These residues and the Fe(II) and 2OG-binding sites are all conserved in Riox2 proteins across species throughout evolution (Additional file 12: Figure S12). Alignment of Riox2 sequences from human, mouse, chicken, zebrafish and Hydra revealed additional aa stretches in the zebra fish and Hydra proteins, which are not present in the other species. The zebra fish Riox2 displayed an additional 30aa stretch between the $\mathrm{JmjC}$ and the dimerization domains (Additional file 12: Figure S12). The Hydra sequence has an extended dimerization domain and an additional 64aa stretch within its WH domain (Additional file 12: Figure S12).

The aforementioned conserved active site and substrate binding residues in Riox 1 and Riox 2 prompted us to look into the sequence homology of their known substrates Rpl8 and Rpl27a in corresponding species. Comparing the Rpl8 and Rpl27a sequences of 39 different eukaryotic species available in the Ribosomal Protein Gene Database (http://ribosome.med.miyazaki-u.ac.jp) [32] revealed that a corresponding $\mathrm{H}$ residue that may be hydroxylated by Riox1 or Riox 2 can be found in each species (data not shown). An alignment of the aa-stretches surrounding the $\mathrm{H}$ hydroxylated by human RIOX1 and RIOX2 showed almost identical aa-sequences for Rpl8 and Rpl27a from ten different species (Additional file 13: Figure S13A,B).

\section{The $\mathrm{N}$-terminal extension domain in Riox 1}

Despite the characteristic domain structure of the ROXs, an obvious difference between Riox 2 and Riox 1 is the $N$-terminal extension present in the Riox1 sequences. This $N$-terminal extension domain has no assigned function yet, but aa 1-31 have been predicted to possess a nuclear localization signal [28]. We further investigated this hypothesis using green fluorescent protein (GFP) fusion reporter experiments. Full-length human RIOX1 tagged with GFP localized predominantly in nucleoli of transfected HeLa cells (Fig. 4a). In contrast, a GFP-tagged deletion mutant lacking aa 1-31 displayed an exclusive cytoplasmatic localisation (Fig. 4b). When we fused the N-terminus of human RIOX1 (aa 1-45) to GFP, the fusion protein localised to the nucleus and accumulated in nucleoli as well (Fig. 4c). We used two different bioinformatic tools (NLSmapper \& NLStradamus) [33, 34] to predict nuclear localization signals (NLS) in Riox1 of several species. In each species, the NLS motive was identified with NLSmapper and NLStradamus tools within the $N$-terminal extension of the analysed Riox1 proteins (Fig. 4d). In contrast, the mechanism of how Riox 2 is directed into the nucleus and/or nucleolus is to our knowledge not known yet. Both NLS prediction tools did not identify corresponding NLSs in all analysed Riox2 protein sequences (data not shown).

\section{Comparative genomic analysis of Riox 1 and Riox 2 across the animal kingdom}

Analyses of the human RIOX1 and RIOX2 genes using the Ensembl genome browser portal revealed 10 exons for human RIOX2. The gene encodes 9 different transcripts and comprises 9 coding exons and alternatively used non-coding 5'-UTR and 3-UTR exons. The largest transcript, RIOX2-001 (ENST00000333396.11), encodes a protein of 465 aa and encompasses a locus of 30,639 base pairs (bp) on human chromosome 3 (Fig. 5a). Surprisingly, the human RIOX1 gene encodes one transcript RIOX1003 (ENST00000304061.7) and is an intronless gene, spanning 2428 bp with 5'- and 3'-UTRs on chromosome 14 (Fig. 5b). Mapping of the characteristic ROX protein domains (JmjC, dimerization, $\mathrm{WH}$ ) to the exon-intron gene structure of RIOX2 revealed that the JmjC domain is encoded by exons $2-4$ and part of exon 5 . RIOX2 exons 5 , 6 and part of exon 7 encode the dimerization domain, whereas the $\mathrm{WH}$ domain is encoded by exons 7, 8, 9 and part of 10 (Fig. 5a). When we compared genomic structures of Riox 1 and Riox 2 orthologues throughout evolution we found that Riox 2 comprises ten (nine coding and one non-coding 3'-UTR) exons in human, mouse and chicken or 9 coding exons in Xenopus, 11 exons in zebra fish and 9 exons in Hydra (Fig. 5c).

To gain additional insights into the evolution of the Riox 1 and Riox2 genes we included additional metazoan species that represent key taxa from the animal kingdom and constructed phylogenetic trees for both genes. Protein sequences encoded by Riox 1 and Riox 2 orthologues were identified in the Ensembl and EnsemblMetazoa genomic resources using the human RIOX1 and RIOX2 protein reference sequences in Protein BLAST (blastp) search routines or the Ensembl evidence-based annotation of orthologues from pre-existing whole-genome pairwise alignments which are available on both Ensembl portals. Obtained protein sequences were validated as being true orthologs using the following criteria: (i) Independent blastp searches with the Homo sapiens RIOX1/RIOX2 and Hydra vulgaris Riox1/Riox2 reference sequences mapped to a single locus in the respective genome with an E-value $<1 \times 10^{-40}$; (ii) Multiple blastp sequence hits ( $>2$ ) were obtained with the human and Hydra query sequence in the same locus; and (iii) The extracted full-length protein sequence followed the characteristic domain structure of ROX proteins. As an additional criterion for correct assignment of Riox 1 orthologs, we also included the presence of the above described $\mathrm{N}$-terminal extension domain in the candidate sequence. All Riox1 ortholog sequences downloaded from the Ensembl portals matched these criteria with the exception of the 


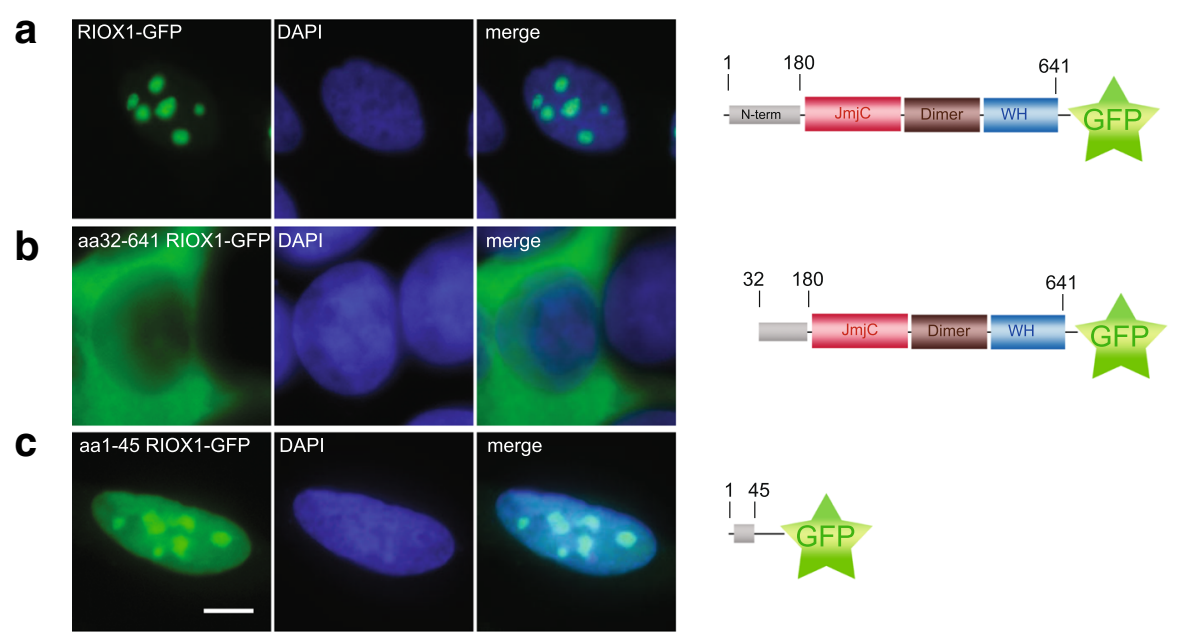

d NLS prediction (RIOX1):

\begin{tabular}{|c|c|}
\hline H. sapiens & $\begin{array}{lr}1 & 51 \\
\text { MDGLQASAGPLERGRPKRRRKPQPHSGSVLALPLRSRKIRKQLRSVVSRMA. } & 1 \\
\text { (aa12-41) } & \end{array}$ \\
\hline M. musculus & 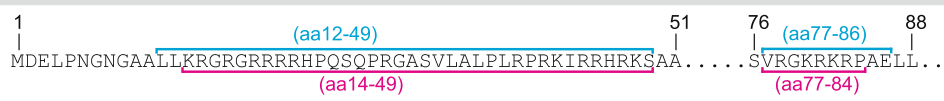 \\
\hline G. gallus & 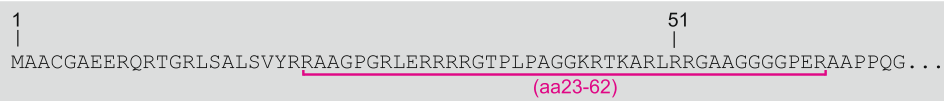 \\
\hline D. rerio & 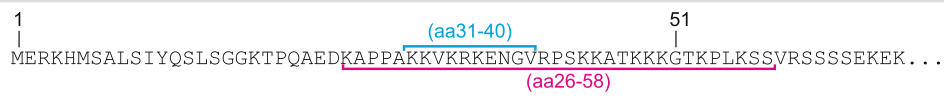 \\
\hline C. elegans & $\begin{array}{c}76 \\
\perp \\
\ldots \text { PKQNGGTKRKHVEVTTQKLENKKPKVEVKKEDEKSKNKKMMKNQNKHTALVQNETSTRSTYFVEE. . } \\
\frac{(\text { aa96-116) }}{(\text { aa80-102) }}\end{array}$ \\
\hline D. melanogaster & $\begin{array}{l}76 \\
\quad \text { I } \\
\ldots \text { KTQSGSAAAAKT'ADRKRRLQAEADANNNDTGKAGKLTKESEATQGARATKRKQARSLGLERTSP. . }\end{array}$ \\
\hline 1. vulgaris & $\begin{array}{l}26 \\
\quad 1 \\
\ldots \text { SPSSKSLNT PSKNGDMKSSNVKKKKLKRNKNEPLNSLRNSIRKKVKKDAHHE ISLKPAENKRKPSV . . . }\end{array}$ \\
\hline
\end{tabular}

Fig. 4 Nuclear localisation of Riox1 and Riox2. The N-terminal extension domain of human RIOX1 has been shown to harbour the nuclear localisation signal (NLS) [28]. a Expression of GFP-tagged full-length RIOX1 in HeLa cells resulted mainly in nucleolar accumulation. b A RIOX1 deletion mutant lacking aa 1-31 localised to the cytoplasm. c Fusion of aa 1-45 of human RIOX1 resulted in nuclear GFP localisation with strong accumulation in the nucleoli. DNA stain: DAPI. Scalebar: 5 m. $\mathbf{d}$ Predictions of NLS in Riox1 of other species with either NLSmapper (blue) or NLStradamus (pink) identified NLS in the N-terminal extension domains of Riox1, whereas for Riox2 no NLS were predicted

Trichoplax adhaerens sequence, which contained a JmjC domain but had neither a dimerization nor a WH domain. Thus, we could not classify this Trichoplax adhaerens ImjC protein as an Riox1 or Riox 2 orthologous gene, however, we included the sequence in the multiple sequence alignments to root the generated phylogenetic trees to a basic metazoan species for which we had sufficient high quality sequence information available on the EnsemblMetazoa portal. The protein phylogenetic analysis of Riox1 orthologs together with the architectures of their genes as annotated in Ensembl reveals that intronless RIOX1/Riox1 genes have evolved independently at least two times in the animal kingdom. Intronless RIOX1/Riox 1 genes are present in the mammalian lineage (humans, mice, and rats), in the marsupial opossum Monodelphis domestica, and in chicken as the representative species of the avian lineage (Fig. 6). Fish, amphibians and other chordates all possess multi-exon genes with up to 15 exons (Latimeria chalumnae) and differently sized and structured non-coding 5'- and 3'-UTR exons (Fig. 6). Within the clade Insecta at least another evolution of an intronless Riox 1 gene must have taken place. We identified intronless loci in Drosophila melanogaster, Drosophila ananassae, and 7 other Drosophila species that encoded intact, full-length open reading frames (ORFs) 


\section{a Human RIOX2 exon structure}

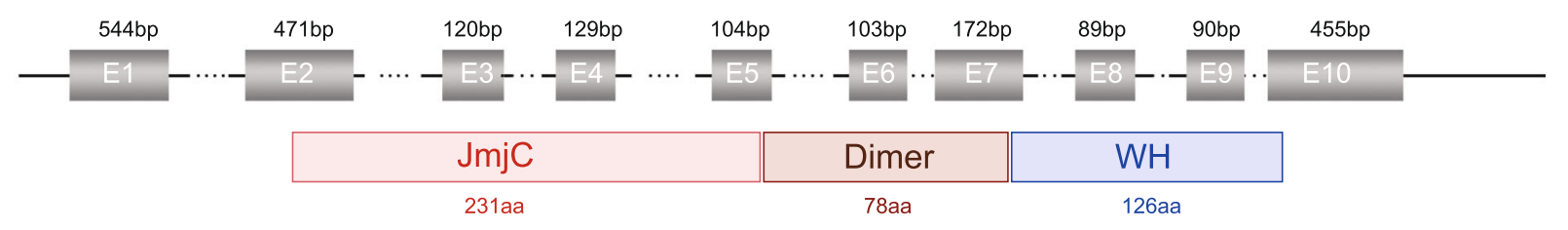

\section{b Human RIOX1 exon structure}

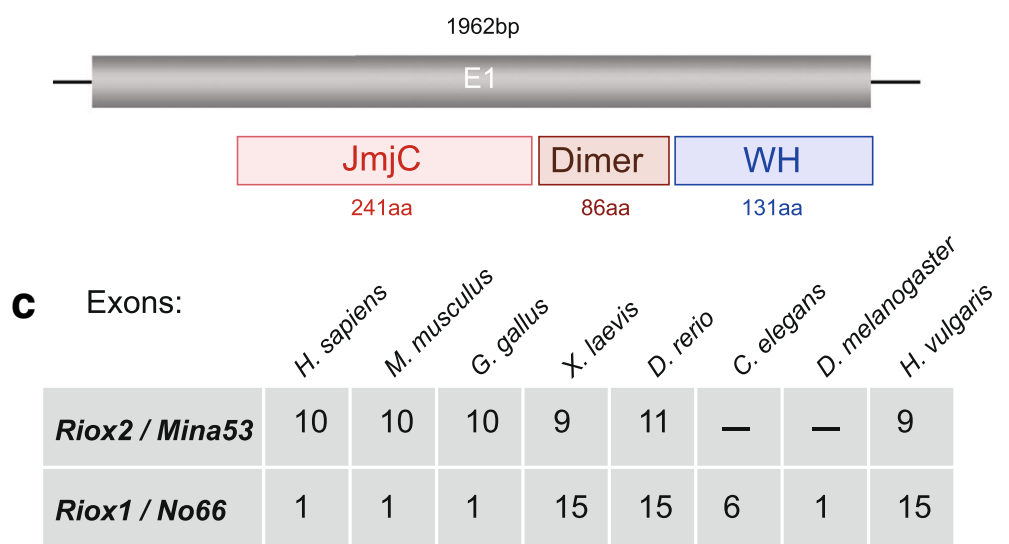

Fig. 5 Riox 1 and Riox2 gene structures. a The human RIOX2 gene exhibits ten exons distributed on chromosome 3. The exon lengths are indicated. The RIOX2 protein domains (JmjC, dimerization, WH) were mapped on the gene. $\mathbf{b}$ The human RIOXI gene is a single exon gene of 1962 bases on chromosome 14. The RIOX1 protein domains (JmjC, dimerization, WH) were mapped on the gene. c Analysis of the genomic structures of Riox2 and Riox1 genes in H. sapiens, M. musculus, G. gallus, X. laevis, D. rerio, C. elegans, D. melanogaster and H. vulgaris with the number of exons are given in the Table. C. elegans and D. melanogaster lack a Riox1 gene. The Riox2 genes of human (Hs), mouse (Mm) and chicken (Gg) exhibit one non-coding exon (5') and nine coding exons

for Riox1 proteins within a size range of 653 to 907 aa (Fig. 6, and data not shown). Importantly, these intronless Riox 1 genes are not clustered within the Insecta clade. Other Drosophila species like D. erecta, D. mojavensis, and D. sechellia have Riox 1 genes that consist of 2 exons and phylogenetically related flies such as Anopheles gambiae, Culex quinquefasciatus, Aedes aegypti, and Belgica antarctica also possess Riox1 genes with two exons. Other representative species of the Insecta clade such as Tribolium castaneum and Atta cephalotes have multi-exon Riox1 genes, however, in contrast to the majority of other analysed metazoan species the number of exons in these insects does not exceed a total number of 6 .

Protein sequence alignments [35] of Riox1 and Riox2 JmjC domains obtained from 49 species and the Escherichia coli (E.coli) ROX protein ycfD and construction of phylogenetic trees using maximum likelihood and bootstrap analysis (data not shown) revealed congruent tree topology. Our phylogenetic study shows a strong node support for a Riox2-JmjC domain branch clearly separating this from Riox1-JmjC domains (Fig. 7). Within the Riox2 branch only closely related taxa (e.g. Ciona) or taxonomic higher ranks (e.g. Chordata) possess high node support making an assumption about the diversification process and possible functions of Riox 2 in different taxa difficult. The strong support for the branching node Riox2 Hydra vulgaris/ecycfD E. coli to the remaining Riox2-possessing species suggests an early invention of Riox2. Interestingly, Riox 2 showed the closest sequence relatedness to the nematode Riox 1 analogs, demonstrating that Riox2 has evolved in the chordata lineage from an ancestral Riox1 gene (Fig. 7). Again strong support for Riox1 Jmjc domains relatedness is given for closely related taxa (e.g. D. rerio + O. latipes, B. mori $+H$. melpomene) or higher taxa ranks (e.g. Mammalia, Chordata, Mollusca). Weak node support (e.g. H. robusta + Chordata, S. purpuratus + L. salmonis/Insecta) indicates that additional research is needed to fully understand the evolution of this gene and its functional diversification.

\section{Discussion}

Although both proteins, Riox1 and Riox 2 are present in Hydra vulgaris, our phylogenetic analyses revealed the absence of Riox 2 in all other investigated invertebrate lineages with the exception of the small phylum Priapulida represented by the marine worm Priapulus caudatus. All 


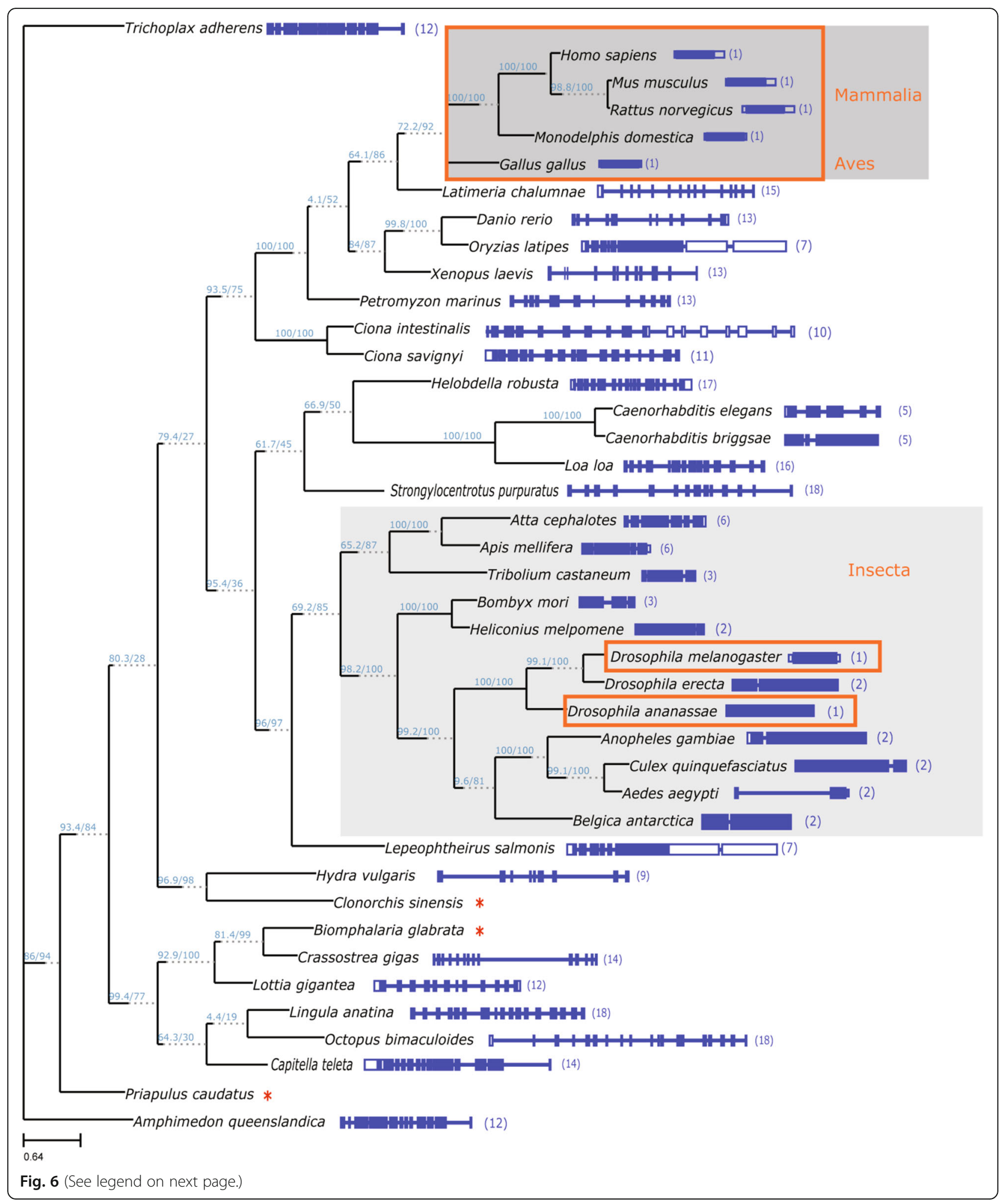


(See figure on previous page.)

Fig. 6 Phylogenetic tree of Riox1 (No66) orthologues proteins and exon-intron architecture of the corresponding protein encoding genes. The tree was inferred through a maximum-likelihood analysis of 41 representative species (IQ-TREE). The tree shown is a consensus tree with $\mathrm{SH}$-like aLRT and ultrafast bootstrap (UFboot) values (numbers in parentheses SH-aLRT support (\%)/ultrafast bootstrap support (\%)) given as branch support values. Good branch support is confirmed with SH-aLRT $>=80 \%$ and UFboot $>=95 \%$. The Tree is unrooted although the outgroup taxon 'Trichoplax' is drawn at root. The scale bar indicates 0.64 substitutions per site. Blue boxes and lines on the right show the gene architectures of the corresponding genes with exons and introns, respectively. Filled boxes represent protein-coding exons, empty boxes represent non-coding 5'- and 3'-UTR exons. Numbers in parentheses indicate total number of exons. Red rectangles encircle single exon, intronless genes present in three different taxa (Mammalia, Aves, and Insecta, grey background shading). Stars indicate species for which completed (non-fragmented) gene architecture annotations are yet not available

analysed Chordata and Echinodermata, and Hyperoartia possess a Riox2 gene. This suggests that at the basis of the Chordata, which are represented by the Tunicata Ciona intestinalis and Ciona savignyi, Riox2 was first evolved from a common ancestral Riox gene. In contrast, Riox 1 is ubiquitously present throughout the animal kingdom. This suggests that Riox 1 is the phylogenetically older gene from which Riox 2 evolved, possibly in two independent events in early Cnidaria (H. vulgaris) and Priapulida ( $P$. caudatus). The sequence homology of the $H$. vulgaris Riox2 JmjC domain to the JmjC domain of the E. coli ycfD protein (Fig. 7) suggests that Riox2 has a ribosomal oxygenase function like its prokaryotic analog [6].

Comparison of the genomic architecture manifested a common exon/intron structure for Riox 2 genes throughout evolution. In all analysed organisms, the Riox 2 gene consists of 9-10 exons. In contrast, Riox 1 genes possess strikingly different gene structures throughout the animal kingdom. All Mammalia and Aves (birds) have intronless Riox1 genes (Fig. 6). Insecta have Riox1 genes with few exons (2 to 6) with some species of Drosophila possessing also single exon genes (Fig. 6).

This suggests two possible scenarios of gene evolution in the Insecta lineage. One possibility is that the Drosophila clade has acquired an intronless Riox 1 gene at the branch point of divergence from other fly species and this intronless gene copy has been maintained in particular Drosophila species. Alternatively, a single exon Riox 1 gene was evolved by retroduplication at the branch point of all Insecta and introns were then independently acquired in different fly subclades with the exception of the Drosophila clade, in which intronless Riox1 genes have been maintained in some but not all Drosophila species. In the latter scenario, an evolutionary selection pressure must have preserved the maintenance of those intronless genes in particular Drosophila species. Reasons for such a preservation of intronless Riox1 loci throughout evolution are unknown but as discussed below different explanations are possible.

Approximately 9\% of all genes in the human genome are intronless [36]. Intronless genes are typically expressed at a lower level compared to intron-containing genes and evolve through retroduplication events in which mRNA transcripts are reverse-transcribed into DNA and inserted into genomes [37-39]. Such retroposition events are an important mechanism for the origination and evolution of new genes from ancestral, parental source genes $[38,40]$. New retropositioned genes may eventually evolve new functions through recruitment of promoter and/or enhancer elements from other regions in the genome and may thereby acquire new spatiotemporal expression patterns, which are different from the ancestral source gene [40]. This can then result in novel tissue- or cell-specific functions of retroposed intronless genes [37]. Interestingly, Riox1 single exon genes are mainly present in taxa with terrestrial lifestyles. Taxa representing animals with predominantly aquatic lifestyles have multiexonic genes with up to 18 exons (Fig. 6). The reason for this divergence in Riox1 gene architectures is unknown but it may be related to oxygen content and physiological requirements in these diverse environments. Riox proteins are members of the JmjC protein superfamily and as such their enzymatic dioxygenase activity highly depends on the concentration and availability of molecular oxygen [11]. Multiexonic genes and their capability to express multiple Riox1 isoforms with different functions might better suit aquatic organisms, that are dependent on dissolved oxygen for respiraton to adapt to different environments. In contrast, intronless genes have generally a narrower expression pattern and are usually also expressed at lower levels compared to multiexonic genes. With human and mouse Riox1 genes we have undertaken a preliminary in silico expression analysis with publically available microarray datasets and expression atlases of primary cells and tissues using the network analysis tool BioLayout/Miru [41, 42]. Searches for Riox1 co-expression networks did not identify defined clusters of co-expressed genes, probably due to the low level and ubiquitous expression pattern of Riox 1 in most analysed cells and tissues (data not shown). However, many intronless genes are known to be associated with stress or immune response induced signalling pathways, which require fast induction of gene and protein expression. Here, the circumvention of complex primary mRNA processing steps such as splicing may insure faster response rates of expression induction. Examples of such intronless genes are members of the heat shock 70 gene family [43], interferon-coding genes [44] and genes encoding $\mathrm{G}$ protein-coupled receptors [45]. Future research may 


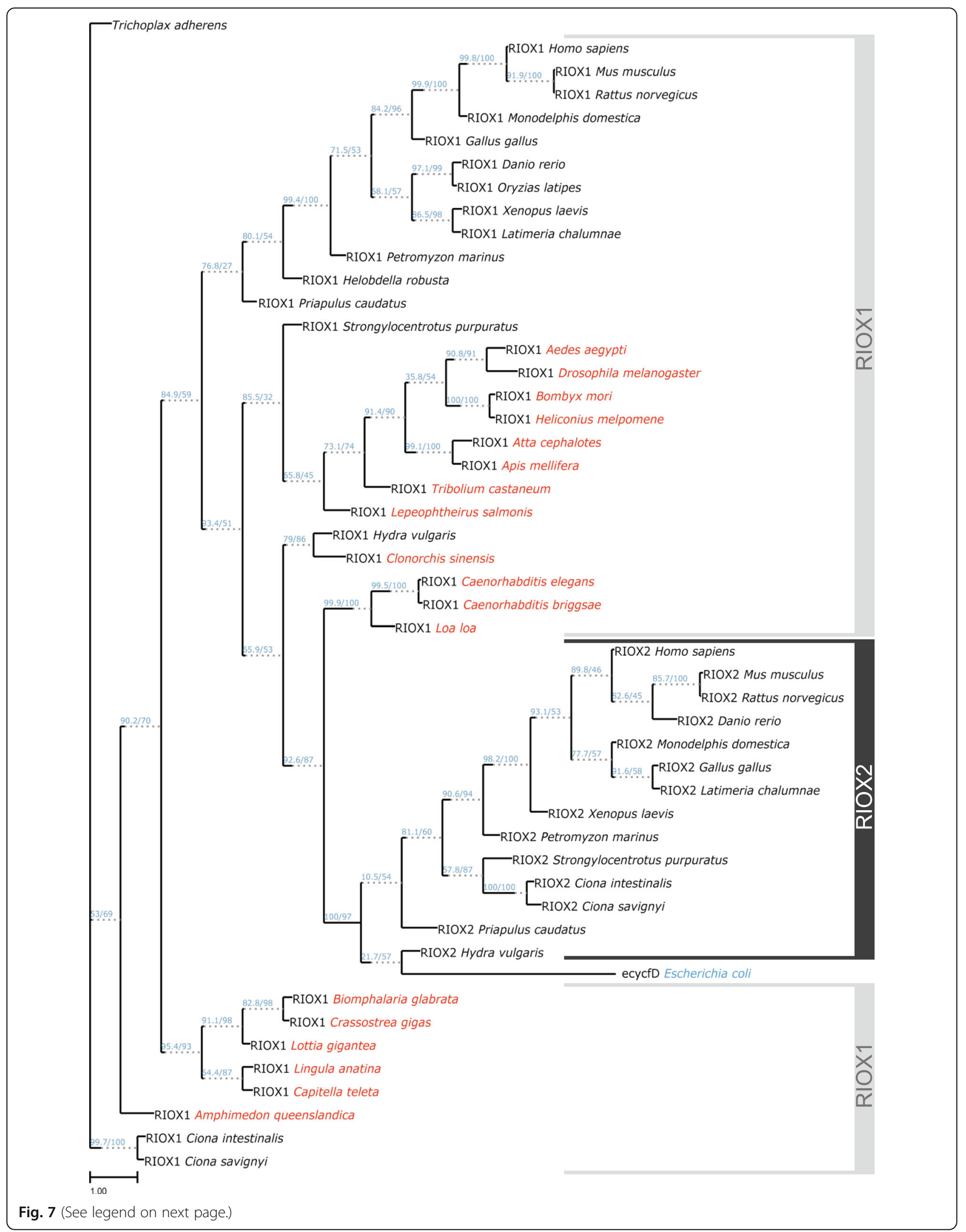


(See figure on previous page.)

Fig. 7 Phylogenetic relationship of Riox1 and Riox2 JmjC domain sequences in Metazoa. Riox1- and Riox2-JmjC domain sequences from species used in this study were extracted from full-length protein sequences, aligned using ClustalW and maximum-likelihood analysis used for tree construction (IQ-TREE). The tree shown is a consensus tree with SH-like aLRT and ultrafast bootstrap (UFboot) values (numbers in parentheses SH-aLRT support (\%)/ultrafast bootstrap support (\%)) given as branch support values. Good branch support is confirmed with SH-aLRT > = 80\% and UFboot > =95\%. The Tree is unrooted although the outgroup taxon 'Trichoplax' is drawn at root. The scale bar indicates 1.00 substitutions per site. The JmjC-domain of the $E$. coli ycfD ribosomal oxygenase (ecycfD) was included in the alignment to analyse its phylogenetic relationship to metazoan Riox1 and Riox2 proteins (indicated in red). Riox2-JmjC domain branch is highlighted with grey background shading to show its separate branch node relationship to Riox1-JmjC domains. Note, Riox2 is also present in Hydra vulgaris and Priapulus caudatus which both possess Riox 1 and Riox2 orthologous genes as invertebrates. Species with a Riox1 gene, but which lack a Riox 2 gene are highlighted in red

identify conditions under which Riox 1 expression is specifically induced and these might be related to stress inducing conditions. This hypothesis is supported by the finding that the closely sequence related paralog of Riox1 and Riox2, Jmjc-1 of Caenorhabditis elegans, is involved in the regulation of an evolutionary conserved stress-response network [46]. In addition, ycfD has been very recently identified in the thermos- and halophilic obligate aerobe Rhodothermus marinus ( $R$. marinus). The marine $R$. marinus of the bacterial phylum Bacteriodetes grows at temperatures around $65{ }^{\circ} \mathrm{C}$ but its ycfD is also able to catalyse Arg hydroxylation of Rpl16 (uL16) [10]. By now ribosomal oxygenases have been identified in bacteria and eukaryotes, though they seem to be not present in archea [16].

Our protein sequence analyses of the ribosomal oxygenases Riox1 and Riox2 in several eukaryotic organisms revealed a conserved protein domain architecture, which initially has been identified by X-ray crystallography in human RIOX2, RIOX1 and the prokaryotic yfcD. However, the lengths of these three domains ( $\mathrm{JmjC}$, dimerization, $\mathrm{WH}$ ) is limited by a range of variation with some exceptions for organisms exhibiting specific extended sequence elements. As genome sequence coverage and qualities of genome assembly still varies among the analysed organisms some of these extended sequence elements might represent annotation mistakes generated by Ensembl's automatic genebuild pipeline. In the cnidarian Hydra vulgaris the Riox2 sequence encodes two extended aa stretches with no corresponding matches in the human sequence. Cloning of the respective Hydra Riox2 from cDNA confirmed the occurrence of those sequence elements. Interestingly, cellular localisation of the Hydra Riox2 is also slightly different to the human protein. While human RIOX2 shows nucleoplasmic localisation with distinct accumulation in nucleoli (Fig. 2) [27, 28], the Hydra orthologue is a nuclear protein but seem to exclude nucleoli (Fig. 3). However, the conserved active site residues imply a fully functional enzyme. Further detailed molecular analyses would be necessary to unravel the enzymatic activity of Hydra Riox2. The original described activity of the human RIOX2 protein was demethylation of histone 3 lysine 9 tri-methylated (H3K9me3) residues [47], however, the evidence for this assignment is controversial [48]. Whether or not
Riox2 might have dual functionality as a ribosomal oxygenase and/or as a histone demethylase needs to be separately investigated in each species which possesses an orthologous gene. Such studies should be further supported with structural approaches, such as those described for another Hydra 2OG oxygenase, Jmjd6 [49-51]. It is likely that throughout evolution Riox 2 proteins have acquired different or additional substrate targets and hydroxylation activities.

\section{Conclusions}

In conclusion, our phylogenetic and genomic analysis of Riox1 and Riox 2 has revealed the maintenance of a unique protein architecture with conservation of active enzymatic sites throughout evolution in the whole animal kingdom. This strongly suggests that both orthologues have a consistent enzymatic function as $\mathrm{Fe}(\mathrm{II})$ and 2OG-dependent dioxygenases with likely ribosomal protein hydroxylation as their main function. However, at the genomic level both orthologs show diversifications in the evolution of their gene architectures and presence or absence of the Riox 2 gene in different taxa. Many higher vertebrates and certain fly species (e.g. Drosophila) possess an intronless Riox 1 orthologue, while Riox 2 is absent in most invertebrates. This suggests that Riox1 is the evolutionary older JmjC-domain-containing protein with ribosomal oxidase function. The more complex gene structure of Riox1 with multiple exons and introns in lower marine metazoans suggests a different, perhaps, more complex regulation of protein expression in these organisms. If this hypothesis is true, that complex Riox 1 gene structures and expression regulation correlates with adaptation to different environments remains to be determined by future research. Differences in expression of protein isoforms and/or subcellular localisations of Riox orthologs in different species, as shown here for Hydra, may be explained by different functional requirements and evolutionary lifestyle adaptation of different taxa.

\section{Methods}

\section{Molecular cloning}

Human full-length and truncated RIOX1 and RIOX2 sequences were sub-cloned into the mammalian expression plasmid pEGFP-N1 (Clontech). Hydra Riox1 and Riox2 
were amplified from cDNA (Hydra vulgaris, total RNA extracted from whole animals, primer: hyNO66_NheF 5'CAGGCTAGCATGAATAACAACAAAGTATCAGC-3', hyNO66_XmaR 5'-GACCCGGGTGTATGGACCAATGGA ACC-3' for Riox1, and hyMina53_NheF CAGGCTAGC ATGGTGAAACGCAAAGGTTC, hyMina53_XmaR GGCCCGGGTTTGATTTCAATCAAATCATCAC, for Riox2, respectively) and sub-cloned into the Hydra eGFP expression plasmid pHotG [31] by using the Nhe1 and Xma1 restriction sites.

\section{Cell culture and transfection}

HeLa cells (ATCC; CCL-2) were cultured in Dulbecco's modified Eagle's medium (DMEM) supplemented with $10 \%$ fetal calf serum (FCS), penicillin $\left(100 \mathrm{Uml}^{-1}\right)$ and streptomycin $\left(100 \mu \mathrm{gml}^{-1}\right)$ at $37{ }^{\circ} \mathrm{C}, 5 \% \mathrm{CO}_{2}$. Cells were transfected with expression constructs using Lipofectamine 2000 (Invitrogen) according to manufacturer's instructions.

\section{Hydra culture}

Hydra vulgaris strain Basel [52] were held in mass culture in hydra medium $(0,1 \mathrm{mM} \mathrm{KCL}, 1 \mathrm{mM} \mathrm{NaCl}$, $0,1 \mathrm{mM} \mathrm{MgSO} 4,1 \mathrm{mM}$ Tris, $1 \mathrm{mM} \mathrm{CaCl}$ ), at a constant temperature of $18{ }^{\circ} \mathrm{C}$ and were fed regularly with freshly hatched Artemia nauplii.

\section{Transfection of Hydra cells}

2,4 mg Gold particles (1,0 $\mu \mathrm{m}$, BioRad) were coated with $10 \mu \mathrm{g}$ plasmid DNA according to instructions of manufacturer. They were introduced into the Hydra cells with a Helios gene gun system (BioRad) as described [31].

\section{Fixation and mounting of Hydra}

Animals were relaxed in $2 \%$ urethane in hydra medium and fixed with $4 \%$ paraformaldehyde (in PBS) at room temperature for one hour. After three washes with PBS, they were counterstained for DNA with DAPI (Sigma, $1 \mu \mathrm{g} / \mathrm{ml}$ ) and mounted on slides with Vectashield mounting medium (Axxora).

\section{Confocal imaging of Hydra}

Light optical sections were acquired with a Leica TCS SP5-2 confocal laser-scanning microscope. Fluorochromes were visualised with the 405 laser with an excitation wavelength of $405 \mathrm{~nm}$ and emission filters 413 to $443 \mathrm{~nm}$ for DAPI. The argon laser with excitation wavelength of $488 \mathrm{~nm}$ and emission filters 496 to $537 \mathrm{~nm}$ was used for GFP. Image resolution was $512 \times 512$ pixel. To obtain an improved signal-to-noise ratio, each section image was averaged from three successive scans.

\section{Immunostaining and microscopy of Hela cells}

HeLa cells were grown to $50-70 \%$ confluence on $18 \mathrm{~mm}$ diameter coverslips. $24 \mathrm{~h}$ post-transfection cells were fixed with $4 \%$ paraformaldehyde (10 min). GFP-expressing cells were stained with $1 \mu \mathrm{g} / \mathrm{ml}$ DAPI and slides mounted in Vectashield. For antibody staining cells were permeabilized after fixation with $1 \%$ Triton X-100 in PBS and subsequently kept in blocking solution for an hour (10\%FCS, $0.2 \%$ Tween-20 in PBS). Primary antibodies: $\alpha$-Riox1 (Mina53, ab169154, Abcam), $\alpha$-Riox1 (NO66, ab113975, Abcam) and $\alpha$-UBF (sc-9131, Santa Cruz). Secondary antibodies: Alexa Fluor 488 chicken anti-mouse (A21200, Thermo Fisher), Alexa Fluor 594 donkey anti-rabbit (A21206, Thermo Fisher). Slides were imaged with a fluorescence microscope Carl Zeiss LSM 510 META.

\section{Ensemble database searches}

Orthologes of Riox 1 and Riox 2 genes were identified using Ensembl (http://www.ensembl.org/info/about/species.html) and EnsemblMetazoa (http://metazoa.ensembl.org/species. html) portals by employing pblast searches. As described in the result section, the genome of each selected species was queried with the human or Hydra vulgaris RIOX1/Riox1 (NP_078920.2 and XP_002157896.3) and ROX2/Riox2 (NP_694822.2 and XP_002167270) protein reference sequences, respectively. For phylogenetic analyses of orthologues proteins only sequences were included in which both, the human and hydra pblast queries, matched to a single locus of the selected species and the identified protein displayed the characteristic domain architecture of Riox1 and Riox 2 proteins as described below in the results section. Species were selected throughout the animal kingdom to represent main taxonomic classes, where available with at least two species per class depending on genome sequence coverage and quality of gene structure annotations. Hydra vulgaris protein sequences and gene annotations for Riox1 and Riox 2 were obtained from https:// metazome.jgi.doe.gov/pz/portal.html.

\section{Multiple-sequence alignments}

FASTA-formatted amino-acid sequences were aligned using the MAFFT 7 tool (http://www.ebi.ac.uk/Tools/ $\mathrm{msa} / \mathrm{mafft} /$ ) provided by the European Bioinformatics Institute (EBI) using the ClustalW algorithm [53]. The resulting multiple aa sequence alignment was used to generate phylogenetic trees.

\section{Construction of phylogenetic trees}

To analyse the phylogenetic relationship of 41 Riox 1 proteins from different species and to infer the evolutionary relationship of Ribosomal oxygenases, the JmjC domain sequences of Riox1 (No66) and Riox2 (Mina53) from 49 species and of ycfD (ecycfD), the ROX protein from E. coli [6], were subjected to a maximum likelihood analysis using the online phylogenetic tool W-IQ-TREE (Version 1.5.4 at http://iqtree.cibiv.univie.ac.at) [54]. In the IQ-Tree webserver the 'Substitution model' and the 
default 'Auto' settings were selected to determine the best-fit substitution model followed by tree construction. Within the 'Branch Support Analysis' the default settings of an ultrafast bootstrap analysis with 1000 replicates was used, with maximum number of iterations set at 1000 and a minimum correlation coefficient of 0.99 and for the 'Single branch tests' the SH-aLRT branch test with 1000 replicates was selected [55].

\section{Bioinformatic prediction of nuclear localisation sites} Nuclear localisation signal searches have been performed with NLS Mapper (http://nls-mapper.iab.keio.ac.jp/cgi-bin/ NLS_Mapper_form.cgi) [34] and NLStradamus (http:// www.moseslab.csb.utoronto.ca/NLStradamus/) [33].

\section{Additional files}

Additional file 1: Protein sequence alignment (Clustal Omega) [35] of RIOX1 (H.sapiens) and Riox1 (M.musculus). The proposed iron-binding motif ( $\mathrm{H} 340, \mathrm{D} 342, \mathrm{H} 405)$ and the 2OG-interacting K355 for the human sequence [16] are indicated in green or blue respectively. (PDF $68 \mathrm{~kb}$ )

Additional file 2: Protein sequence alignment (Clustal Omega) [35] of RIOX1 (H.sapiens) and Riox1 (G.gallus). The proposed iron-binding motif ( $\mathrm{H} 340, \mathrm{D} 342, \mathrm{H} 405)$ and the 2OG-interacting K355 for the human sequence [16] are indicated in green or blue respectively. (PDF $106 \mathrm{~kb}$ )

Additional file 3: Protein sequence alignment (Clustal Omega) [35] of RIOX1 (H.sapiens) and Riox1 (D.rerio). The proposed iron-binding motif (H340, D342, H405) and the 2OG-interacting K355 for the human sequence [16] are indicated in green or blue respectively. (PDF $106 \mathrm{~kb}$ )

Additional file 4: Protein sequence alignment (Clustal Omega) [35] of RIOX1 (H.sapiens) and Riox1 (D.melanogaster). The proposed iron-binding motif (H340, D342, H405) and the 2OG-interacting K355 for the human sequence [16] are indicated in green or blue respectively. (PDF $103 \mathrm{~kb}$ )

Additional file 5: Protein sequence alignment (Clustal Omega) [35] of RIOX1 (H.sapiens) and Riox1 (C.elegans). The proposed iron-binding motif ( $\mathrm{H340}, \mathrm{D} 342, \mathrm{H} 405)$ and the 2OG-interactingK355 for the human sequence [16] are indicated in green or blue respectively. (PDF $103 \mathrm{~kb}$ )

Additional file 6: Protein sequence alignment (Clustal Omega) [35] of RIOX2 (H.sapiens) and Riox2 (M.musculus). The proposed iron-binding motif (H179, D181, H240) and the 2OG-interacting K194 for the human sequence [16] are indicated in green or blue respectively. (PDF $68 \mathrm{~kb}$ )

Additional file 7: Protein sequence alignment (Clustal Omega) [35] of RIOX2 (H.sapiens) and Riox2 (G.gallus). The proposed iron-binding motif ( $\mathrm{H} 179, \mathrm{D} 181, \mathrm{H} 240)$ and the 2OG-interacting K194 for the human sequence [16] are indicated in green or blue respectively. (PDF $67 \mathrm{~kb}$ )

Additional file 8: Protein sequence alignment (Clustal Omega) [35] of RIOX2 (H.sapiens) and Riox2 (D.rerio). The proposed iron-binding motif $(\mathrm{H} 179, \mathrm{D} 181, \mathrm{H} 240)$ and the 2OG-interacting K194 for the human sequence [16] are indicated in green or blue respectively. (PDF $68 \mathrm{~kb}$ )

Additional file 9: Nucleotide sequence (open reading frame) and corresponding aa sequence of Riox2 (H.vulgaris). Protein sequence alignment (Clustal Omega) [35] of RIOX2 (H.sapiens) and Riox2 (H.vulgaris). The proposed iron-binding motif (H179, D181, H240) and the 2OG-interacting K194 for the human sequence [16] are indicated in green or blue respectively. An additional stretch of charged aa in the Hydra sequence is highlighted in red. (PDF $71 \mathrm{~kb}$ )

Additional file 10: Nucleotide sequence (open reading frame) and corresponding aa sequence of Riox1 (H.vulgaris). Protein sequence alignment (Clustal Omega) [35] of RIOX1 (H.sapiens) and Riox1 (H.vulgaris). The proposed iron-binding motif ( $\mathrm{H340}, \mathrm{D} 342, \mathrm{H} 405)$ and the 2OG-interacting K355 for the human sequence [16] are indicated in green or blue respectively. (PDF $71 \mathrm{~kb}$ )
Additional file 11: Clustal omega alignment of Riox1 protein sequences from C.elegans, D.melanogaster, H.vulgaris, zebrafish, chicken, mouse and human. The protein domains JmjC (red), dimerization (brown) and winged-helix (blue) are indicated based on the human sequence [16]. Lengths of the individual $\mathrm{N}$-terminal extension domains are indicated (grey). The prospective iron-binding motif $\mathrm{HxD} \ldots \mathrm{H}$ (green) and the $2 \mathrm{OG}$ C5-carboxylate-binding residue ( $\mathrm{K}$, purple) are conserved in all species. Crystal structure analysis of human RIOX1 with substrate Rpl8 identified R297, Y328 and S421 residues of human RIOX1 involved in Rpl8 peptide binding [16] (red). (PDF $93 \mathrm{~kb}$ )

Additional file 12: Clustal omega alignment of Riox2 protein sequences from H.vulgaris, zebrafish, chicken, mouse and human. The protein domains JmjC (red), dimerization (brown) and winged-helix (blue) are indicated based on the human sequence [16]. The prospective iron-binding motif $\mathrm{HxD} \ldots \mathrm{H}$ (green) and the $20 \mathrm{G} \mathrm{C5-carboxylate-binding} \mathrm{residue} \mathrm{(K,} \mathrm{purple)} \mathrm{are} \mathrm{conserved}$ in all species. Crystal structure analysis of human RIOX2 with substrate Rpl27a identified the aa N101, Q136, Q139, N165 and S257 of human RIOX2 involved in Rpl27a peptide binding [16] (red). (PDF $90 \mathrm{~kb})$

Additional file 13: Clustal omega alignment of Rpl8 (A) and Rpl27a (B) protein sequences from C. intestinalis, H. vulgaris, C. elegans, A. melifera, fruit fly, zebrafish, $X$. laevis, chicken, mouse and human. The protein sequence corresponds to the aa 208-224 of human RPL8 (A) and aa 30-51 of human RPL27A (B). Both aa-stretches harbour the H-residues, which get hydroxylated by human RIOX1 (H216 in RPL8) and RIOX2 (H39 in RPL27A). Sequences highlighted in red are species where no Riox 2 gene has been identified. (PDF $62 \mathrm{~kb})$

\section{Abbreviations}

2OG: 2-oxoglutarate; D: Aspartate; E: Glutamate; G: Glycine; GFP: Green fluorescent protein; H: Histidine; JmjC: Jumonji C; K: Lysine; N: Asparagine; NLS: Nuclear localisation signal; NoLS: Nucleolar localisation signal; ORF: Open reading frame; Q: Glutamine; ROX: Ribosomal oxygenase; Th2: T helper 2; UTR: Untranslated region; WH: Winged-helix; Y: Tyrosine

\section{Acknowledgements}

The authors thank Prof Angelika Böttger, Prof Charles N David and Dr. Joel Schick for support and advice.

\section{Funding}

This work was supported by the institute strategic grant funding from the Biotechnology and Biological Sciences Research Council (BBSRC, UK; to AL and EWL) and the Deutsche Forschungsgemeinschaft (DFG BO1748/12-1 to JM). Funding bodies had no impact on the design of the study and collection, analysis, and interpretation of data and in writing the manuscript.

\section{Availability of data and materials}

The datasets analysed during the current study are available in the Ensembl database (http://www.ensembl.org/info/about/species.html), the EnsemblMetazoa database (http://metazoa.ensembl.org/species.html) or the Hydra Genome Database (https://metazome.jgi.doe.gov/pz/portal.html). The datasets used and/or analysed during the current study are available from the corresponding author on reasonable request.

\section{Authors' contributions}

AW designed the study. KEB, AL and AW performed the genomic analyses. EWL performed the phylogenetic analyses. KB produced the deletion constructs. KEB, KB and AF analysed proteins in human cells. KEB cloned the Hydra sequences. KEB and JM performed the experiments in Hydra. AL and AW wrote the manuscript. All authors read and approved the final manuscript.

\section{Ethics approval and consent to participate}

Not applicable.

\section{Consent for publication}

Not applicable.

\section{Competing interests}

The authors declare no competing or financial interests. 


\section{Publisher's Note}

Springer Nature remains neutral with regard to jurisdictional claims in published maps and institutional affiliations.

\begin{abstract}
Author details
Institute of Molecular Toxicology and Pharmacology, Helmholtz Zentrum München-German Research Center for Environmental Health, Ingolstädter Landstrasse 1, 85764 Neuherberg, Germany. ${ }^{2}$ Department of Biology II, Ludwig Maximillians University, Munich, Großhaderner Strasse 2, 82152 Planegg-, Martinsried, Germany. ${ }^{3}$ Research Unit Analytical Pathology, Helmholtz Zentrum München, German Research Center for Environmental Health GmbH, Ingolstädter Landstr. 1, 85764 Neuherberg, Germany. ${ }^{4}$ The Roslin Institute and Royal (Dick) School of Veterinary Studies, University of Edinburgh, Edinburgh, UK. ${ }^{5}$ Present address: Max-Planck-Society, Administrative Headquarters, Hofgartenstr. 8, 80539 Munich, Germany.
\end{abstract}

\section{Received: 9 October 2017 Accepted: 7 June 2018}

\section{Published online: 19 June 2018}

\section{References}

1. Crick F. Central dogma of molecular biology. Nature. 1970;227(5258):561-3.

2. Rissland OS. The organization and regulation of mRNA-protein complexes. Wiley Interdiscip Rev RNA. 2017;8:e1369.

3. Roundtree IA, He C. RNA epigenetics-chemical messages for posttranscriptional gene regulation. Curr Opin Chem Biol. 2016;30:46-51.

4. Shi Z, Barna M. Translating the genome in time and space: specialized ribosomes, RNA regulons, and RNA-binding proteins. Annu Rev Cell Dev Biol. 2015;31:31-54.

5. Xue S, Barna M. Specialized ribosomes: a new frontier in gene regulation and organismal biology. Nat Rev Mol Cell Biol. 2012;13(6):355-69.

6. Ge W, Wolf A, Feng T, Ho CH, Sekirnik R, Zayer A, Granatino N, Cockman ME, Loenarz C, Loik ND, et al. Oxygenase-catalyzed ribosome hydroxylation occurs in prokaryotes and humans. Nat Chem Biol. 2012;8(12):960-2.

7. Katz MJ, Acevedo JM, Loenarz C, Galagovsky D, Liu-Yi P, Perez-Pepe M, Thalhammer A, Sekirnik R, Ge W, Melani M et al: Sudestada1, a Drosophila ribosomal prolyl-hydroxylase required for mRNA translation, cell homeostasis, and organ growth Proceedings of the National Academy of Sciences of the United States of America 2014

8. Loenarz C, Sekirnik R, Thalhammer A, Ge W, Spivakovsky E, Mackeen MM, McDonough MA, Cockman ME, Kessler BM, Ratcliffe PJ, et al. Hydroxylation of the eukaryotic ribosomal decoding center affects translational accuracy. Proc Natl Acad Sci U S A. 2014;111(11):4019-24.

9. Singleton RS, Liu-Yi P, Formenti F, Ge W, Sekirnik R, Fischer R, Adam J, Pollard PJ, Wolf A, Thalhammer A, et al. OGFOD1 catalyzes prolyl hydroxylation of RPS23 and is involved in translation control and stress granule formation. Proc Natl Acad Sci U S A. 2014;111(11):4031-6.

10. Sekirnik R, Wilkins SE, Bush J, Tarhonskaya H, Munzel M, Hussein A, Flashman E, Mohammed S, McDonough MA, Loenarz C, et al. YcfDRM is a thermophilic oxygen-dependent ribosomal protein uL16 oxygenase. Extremophiles. 2018;22(3):553-62.

11. Aik W, McDonough MA, Thalhammer A, Chowdhury R, Schofield CJ. Role of the jelly-roll fold in substrate binding by 2-oxoglutarate oxygenases. Curr Opin Struct Biol. 2012;22(6):691-700.

12. Bottger A, Islam MS, Chowdhury R, Schofield CJ, Wolf A. The oxygenase Jmjd6-a case study in conflicting assignments. The Biochemical journal. 2015;468(2):191-202

13. Kooistra SM, Helin K. Molecular mechanisms and potential functions of histone demethylases. Nat Rev Mol Cell Biol. 2012;13(5):297-311.

14. Rasmussen KD, Helin K. Role of TET enzymes in DNA methylation, development, and cancer. Genes Dev. 2016;30(7):733-50.

15. Webb JD, Coleman ML, Pugh CW. Hypoxia, hypoxia-inducible factors (HIF), HIF hydroxylases and oxygen sensing. Cell Mol Life Sci. 2009;66(22):3539-54.

16. Chowdhury R, Sekirnik R, Brissett NC, Krojer T, Ho CH, Ng SS, Clifton IJ, Ge W, Kershaw NJ, Fox GC, et al. Ribosomal oxygenases are structurally conserved from prokaryotes to humans. Nature. 2014;510(7505):422-6.

17. Tsuneoka M, Koda Y, Soejima M, Teye K, Kimura H. A novel myc target gene, mina53, that is involved in cell proliferation. J Biol Chem. 2002;277(38):35450-9.

18. Zhang Y, Lu Y, Yuan BZ, Castranova V, Shi X, Stauffer JL, Demers LM, Chen F. The human mineral dust-induced gene, mdig, is a cell growth regulating gene associated with lung cancer. Oncogene. 2005;24(31):4873-82.
19. Thakur C, Lu Y, Sun J, Yu M, Chen B, Chen F. Increased expression of mdig predicts poorer survival of the breast cancer patients. Gene. 2014;535(2):218-24.

20. Suzuki C, Takahashi K, Hayama S, Ishikawa N, Kato T, Ito T, Tsuchiya E, Nakamura Y, Daigo Y. Identification of Myc-associated protein with JmjC domain as a novel therapeutic target oncogene for lung cancer. Mol Cancer Ther. 2007:6(2):542-51.

21. Nishizawa Y, Nishida N, Konno M, Kawamoto K, Asai A, Koseki J, Takahashi $\mathrm{H}$, Haraguchi N, Nishimura J, Hata T, et al. Clinical significance of histone Demethylase NO66 in invasive colorectal Cancer. Ann Surg Oncol. 2017; 24(3):841-9.

22. Okamoto $M$, Van Stry $M$, Chung L, Koyanagi M, Sun $X$, Suzuki Y, Ohara O, Kitamura H, Hijikata A, Kubo M, et al. Mina, an II4 repressor, controls T helper type 2 bias. Nat Immunol. 2009;10(8):872-9.

23. Pillai MR, Lian S, Bix M. Mina: a Th2 response regulator meets TGFbeta. Curr Opin Immunol. 2014:31:38-43.

24. Sinha KM, Yasuda H, Coombes MM, Dent SY, de Crombrugghe B. Regulation of the osteoblast-specific transcription factor Osterix by N066, a Jumonji family histone demethylase. EMBO J. 2010;29(1):68-79.

25. Chen Q, Sinha K, Deng JM, Yasuda H, Krahe R, Behringer RR, de Crombrugghe B. Mesenchymal deletion of histone Demethylase NO66 in mice promotes bone formation. J Bone Miner Res. 2015;30(9):1608-17.

26. Chen Q, Zhang L, de Crombrugghe B, Krahe R. Mesenchyme-specific overexpression of nucleolar protein 66 in mice inhibits skeletal growth and bone formation. FASEB J. 2015;29(6):2555-65.

27. Eilbracht J, Kneissel S, Hofmann A, Schmidt-Zachmann MS. Protein NO52-a constitutive nucleolar component sharing high sequence homologies to protein NO66. Eur J Cell Biol. 2005;84(2-3):279-94.

28. Eilbracht J, Reichenzeller M, Hergt M, Schnolzer M, Heid H, Stohr M, Franke WW, Schmidt-Zachmann MS. NO66, a highly conserved dual location protein in the nucleolus and in a special type of synchronously replicating chromatin. Mol Biol Cell. 2004;15(4):1816-32.

29. Leclere L, Copley RR, Momose T, Houliston E. Hydrozoan insights in animal development and evolution. Curr Opin Genet Dev. 2016;39:157-67.

30. Chapman JA, Kirkness EF, Simakov O, Hampson SE, Mitros T, Weinmaier T, Rattei T, Balasubramanian PG, Borman J, Busam D, et al. The dynamic genome of Hydra. Nature. 2010;464(7288):592-6.

31. Bottger A, Alexandrova O, Cikala M, Schade M, Herold M, David CN. GFP expression in Hydra: lessons from the particle gun. Dev Genes Evol. 2002;212(6):302-5.

32. Nakao A, Yoshihama M, Kenmochi N. RPG: the ribosomal protein gene database. Nucleic Acids Res. 2004;32(Database issue):D168-70.

33. Nguyen Ba AN, Pogoutse A, Provart N, Moses AM. NLStradamus: a simple hidden Markov model for nuclear localization signal prediction. BMC Bioinformatics. 2009:10:202.

34. Kosugi S, Hasebe M, Tomita M, Yanagawa H. Systematic identification of cell cycle-dependent yeast nucleocytoplasmic shuttling proteins by prediction of composite motifs. Proc Natl Acad Sci U S A. 2009;106(25):10171-6.

35. Sievers F, Wilm A, Dineen D, Gibson TJ, Karplus K, Li W, Lopez R, McWilliam $H$, Remmert M, Soding J, et al. Fast, scalable generation of high-quality protein multiple sequence alignments using Clustal omega. Mol Syst Biol. 2011;7:539.

36. Jorquera R, Ortiz R, Ossandon F, Cardenas JP, Sepulveda R, Gonzalez C, Holmes DS. SinEx DB: a database for single exon coding sequences in mammalian genomes. Database. 2016;2016. article ID baw095.

37. Grzybowska EA. Human intronless genes: functional groups, associated diseases, evolution, and mRNA processing in absence of splicing. Biochem Biophys Res Commun. 2012;424(1):1-6.

38. Kaessmann H. Origins, evolution, and phenotypic impact of new genes. Genome Res. 2010;20(10):1313-26.

39. Shabalina SA, Ogurtsov AY, Spiridonov AN, Novichkov PS, Spiridonov NA, Koonin EV. Distinct patterns of expression and evolution of intronless and intron-containing mammalian genes. Mol Biol Evol. 2010;27(8):1745-9.

40. Carelli FN, Hayakawa T, Go Y, Imai H, Warnefors M, Kaessmann H. The life history of retrocopies illuminates the evolution of new mammalian genes. Genome Res. 2016;26(3):301-14.

41. Theocharidis A, van Dongen S, Enright AJ, Freeman TC. Network visualization and analysis of gene expression data using BioLayout express(3D). Nat Protoc. 2009:4(10):1535-50.

42. Mabbott NA, Baillie JK, Brown H, Freeman TC, Hume DA. An expression atlas of human primary cells: inference of gene function from coexpression networks. BMC Genomics. 2013;14:632. 
43. Maquat LE, Li X. Mammalian heat shock p70 and histone H4 transcripts, which derive from naturally intronless genes, are immune to nonsensemediated decay. RNA. 2001;7(3):445-56.

44. Krause CD. Intron loss in interferon genes follows a distinct set of stages, and may confer an evolutionary advantage. Cytokine. 2016;83:193-205.

45. Markovic $\mathrm{D}$, Challiss RA. Alternative splicing of $\mathrm{G}$ protein-coupled receptors: physiology and pathophysiology. Cell Mol Life Sci. 2009;66(20):3337-52.

46. Kirienko NV, Fay DS. SLR-2 and JMJC-1 regulate an evolutionarily conserved stress-response network. EMBO J. 2010;29(4):727-39.

47. Lu Y, Chang Q, Zhang Y, Beezhold K, Rojanasakul Y, Zhao H, Castranova V, Shi $X$, Chen F. Lung cancer-associated JmjC domain protein mdig suppresses formation of tri-methyl lysine 9 of histone H3. Cell Cycle. 2009;8(13):2101-9.

48. Williams ST, Walport LJ, Hopkinson RJ, Madden SK, Chowdhury R, Schofield $\mathrm{CJ}$, Kawamura A. Studies on the catalytic domains of multiple JmjC oxygenases using peptide substrates. Epigenetics. 2014:9(12):1596-603.

49. Cikala M, Alexandrova O, David CN, Proschel M, Stiening B, Cramer P, Bottger A. The phosphatidylserine receptor from Hydra is a nuclear protein with potential Fe(II) dependent oxygenase activity. BMC Cell Biol. 2004;5:26.

50. Mantri M, Krojer T, Bagg EA, Webby CJ, Butler DS, Kochan G, Kavanagh KL, Oppermann U, McDonough MA, Schofield CJ. Crystal structure of the 2oxoglutarate- and Fe(II)-dependent lysyl hydroxylase JMJD6. J Mol Biol. 2010:401(2):211-22.

51. Webby CJ, Wolf A, Gromak N, Dreger M, Kramer H, Kessler B, Nielsen ML, Schmitz C, Butler DS, Yates JR 3rd, et al. Jmjd6 catalyses lysyl-hydroxylation of U2AF65, a protein associated with RNA splicing. Science. 2009;325(5936):90-3.

52. Hemmrich G, Anokhin B, Zacharias H, Bosch TC. Molecular phylogenetics in Hydra, a classical model in evolutionary developmental biology. Mol Phylogenet Evol. 2007;44(1):281-90.

53. Katoh K, Standley DM. MAFFT multiple sequence alignment software version 7: improvements in performance and usability. Mol Biol Evol. 2013:30(4):772-80

54. Trifinopoulos J, Nguyen LT, von Haeseler A, Minh BQ. W-IQ-TREE: a fast online phylogenetic tool for maximum likelihood analysis. Nucleic Acids Res. 2016;44(W1):W232-5.

55. Guindon S, Dufayard JF, Lefort V, Anisimova M, Hordijk W, Gascuel O. New algorithms and methods to estimate maximum-likelihood phylogenies: assessing the performance of PhyML 3.0. Syst Biol. 2010;59(3):307-21.

\section{Ready to submit your research? Choose BMC and benefit from:}

- fast, convenient online submission

- thorough peer review by experienced researchers in your field

- rapid publication on acceptance

- support for research data, including large and complex data types

- gold Open Access which fosters wider collaboration and increased citations - maximum visibility for your research: over $100 \mathrm{M}$ website views per year

At BMC, research is always in progress.

Learn more biomedcentral.com/submissions 